\title{
Clinical Analysis of Bilateral Thalamic Infarction Caused by Percheron Artery Occlusion
}

\author{
Peng Chen' \\ Mei-Mei Hao ${ }^{2}$ \\ Yong Chen ${ }^{3}$ \\ Hong Zhang ${ }^{4}$ \\ Zhe Wang ${ }^{5}$ \\ Bin Zhao' \\ Yani Xue' \\ Yumei Chai ${ }^{\prime}$ \\ Yong-Feng Huang' \\ Jiang Zhu'
}

'Department of Neurology, The First Hospital of Yulin, Yulin, People's Republic of China; ${ }^{2}$ Department of Neurology,

Yan'an People's Hospital, Yan'an, People's Republic of China; ${ }^{3}$ Department of Endoscopy Center, Shaanxi Cancer Hospital, Xi'an, Shannxi Province, People's Republic of China; ${ }^{4}$ Department of Vascular Surgery, Shaanxi Provincial People's Hospital, Xi'an, Shannxi Province, People's Republic of China; ${ }^{5}$ Department of General Practice, Second Affiliated Hospital of Xi'an Jiaotong University, Xi'an, Shannxi Province, People's Republic of China
Correspondence: Jiang Zhu Department of Neurology, The First Hospital of Yulin, No. 93, Yuxi Street, Gaoxin District, Yulin, Shaanxi, 719000,

People's Republic of China

Email 13709204858@I63.com

\begin{abstract}
The Percheron artery (artery of Percheron, AOP) is a rare variant vessel. Its acute occlusion can cause a bilateral symmetrical thalamic stroke; typical symptoms of bilateral paramedian thalamic infarcts due to occlusion of AOP are vertical gaze palsy, memory impairment, confusion, drowsiness, hypersomnolence, or coma. We present the MR imaging findings in two cases with cerebral infarction caused by Percheron artery occlusion. Due to the difficulty in the diagnosis of acute Percheron arterial infarction, early conservative treatment is used. The prognosis of the disease is poor, with few patients completely rehabilitating. Therefore, clinicians must understand the characteristics of the disease, provide early diagnosis and administer timely and effective treatment to reduce the patient's disability rate and fatality rate and therefore improve the quality of life of patients. The patient's prognosis has extraordinary significance.
\end{abstract}

Keywords: thalamic infarcts, artery of Percheron, MR imaging

\section{Background}

The thalamus and midbrain have a complex blood supply system. They receive blood mainly from the vertebrobasilar artery system, supplemented by the internal carotid artery system. ${ }^{1,2}$ The blood supply of the thalamus has four main parts: the tuberothalamic artery, thalamic geniculate artery, paramedian posterior thalamoperforating artery, and posterior choroidal artery. ${ }^{3}$ Percheron described three possible variations involving the paramedian thalamicmesencephalic arterial supply: small branches arising from both P1 segments, an asymmetrical common trunk arising from a P1 segment (this variation is called the artery of Percheron), or an arterial arcade emanating from an artery bridging the two P1 segments. ${ }^{4}$ The Percheron artery was first proposed in 1973. It is a rare anatomical variation where a single thalamic perforating artery originates from the proximal posterior cerebral artery (P1 segment) and supplies the bilateral parathalamus and the rostral mesencephalon. If this artery is occluded, bilateral paramedian thalamic infarction occurs. ${ }^{5}$ Typical symptoms of bilateral paramedian thalamic infarcts due to occlusion of AOP are vertical gaze palsy, memory impairment, confusion, drowsiness, hypersomnolence, or coma. $^{6}$

Patients with bilateral paramedian thalamic infarcts accompanied by rostral midbrain lesions also have hemiplegia, cerebellar ataxia, movement dysfunctions, and oculomotor deficits. ${ }^{7}$ We present the MR imaging findings in two cases with cerebral infarction caused by Percheron artery occlusion. 


\section{Case I}

A 57-year-old male was admitted to the emergency room after being unconscious for two days. His children reported that two days ago, the patient suddenly had dizziness and blurred vision when working in the morning and then fell to the ground and became unconscious, which lasted for approximately 6 hours. He was immediately admitted to the local county hospital. The next day, head CT diagnosis indicated low-density lesions in the bilateral thalamus. Due to limited medical resources at the local county hospital, the patient was transferred to the Department of Neurology of our hospital. A MRI examination was conducted immediately, which suggested bilateral thalamic acute infarction (Figure 1). The patient had had hypertension for 4 years. The patients had no bad habits, such as smoking or drinking, no history of drugs or vaccination, and no history of exposure to toxic substances. Physical examination showed that he was in a drowsy state. Neurological examination revealed the left pupil at $7 \mathrm{~mm}$ and the right pupil at $5 \mathrm{~mm}$ (Figure 2), bilateral pupil reflex to light disappeared, binocular vertical gaze palsy, bilateral Babinski signs were suspicious positive, the NIHSS score was 6 points, and the GCS score was 13 points. The haematology test revealed a low-density lipoprotein concentration of 3.31 mmol/L. Other haematology tests showed no abnormalities. The electrocardiogram was normal, echocardiography showed no abnormality, magnetic resonance angiography was normal (Figure 3), and digital subtraction angiography was normal. The patient gradually recovered after a week in the hospital. During hospitalization, the patient was monitored and underwent regulation of blood pressure, anti-platelet aggregation, stabilization of plaque, improvement of circulation, elimination of oxygen free radicals and other treatments, and the clinical symptoms significantly improved. The patient was admitted to the hospital with a NIHSS score of 6 points, which dropped to 2 points after treatment. The patient was admitted to the hospital with a GCS score of 13 points, which
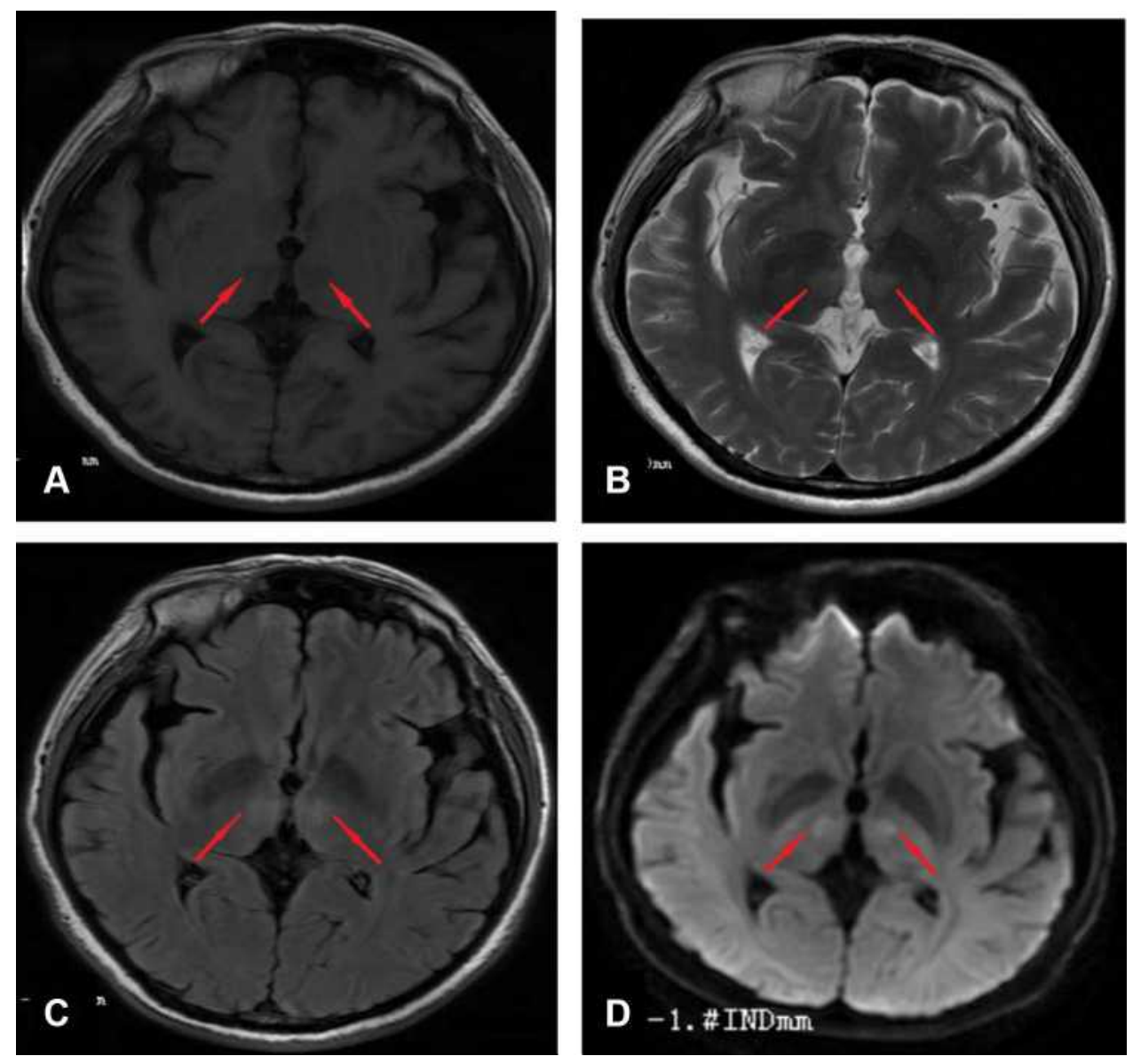

Figure I (A) Magnetic resonance TI-weighted imaging. Images show abnormal low signal bilateral paramedian thalamic lesions (arrows). (B) Magnetic resonance T2weighted imaging. Images show abnormal hyperintense bilateral paramedian thalamic lesions (arrows). (C) Fluid attenuated inversion recovery echo MRI. Images show abnormal hyperintense bilateral paramedian thalamic lesions (arrows). (D) Diffusion-weighted axial MR showing diffusion restriction of the bilateral paramedian thalamus (arrows). 

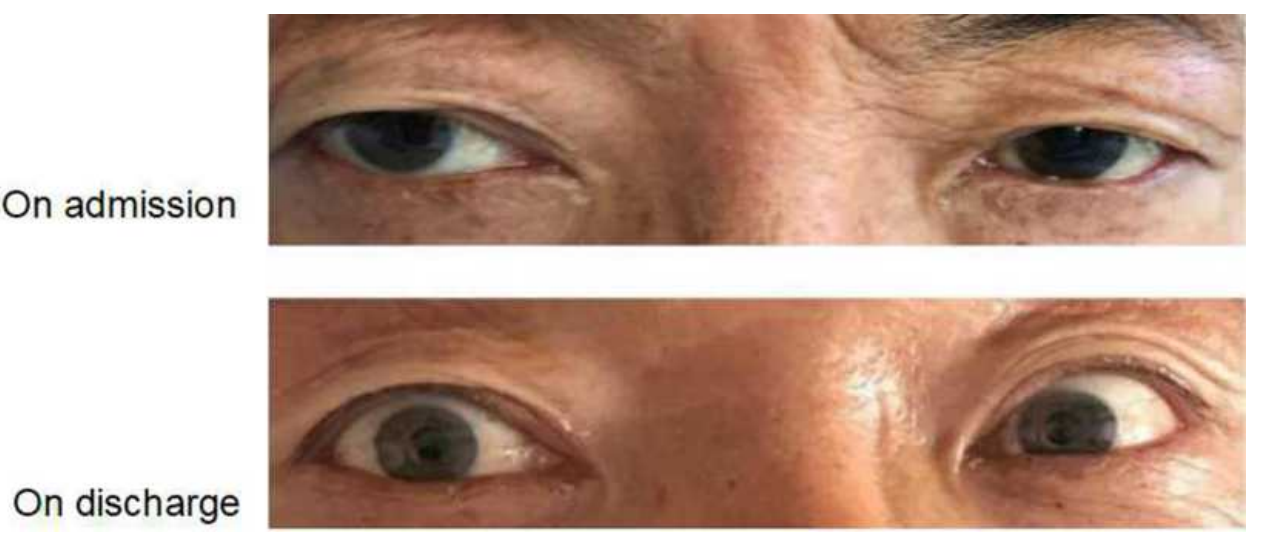

Figure 2 The pupils of the patient were not large at the time of admission; the left pupil was $7 \mathrm{~mm}$, and the right pupil was 5 mm. The patient's bilateral pupils were equally large and round when he was discharged from the hospital.

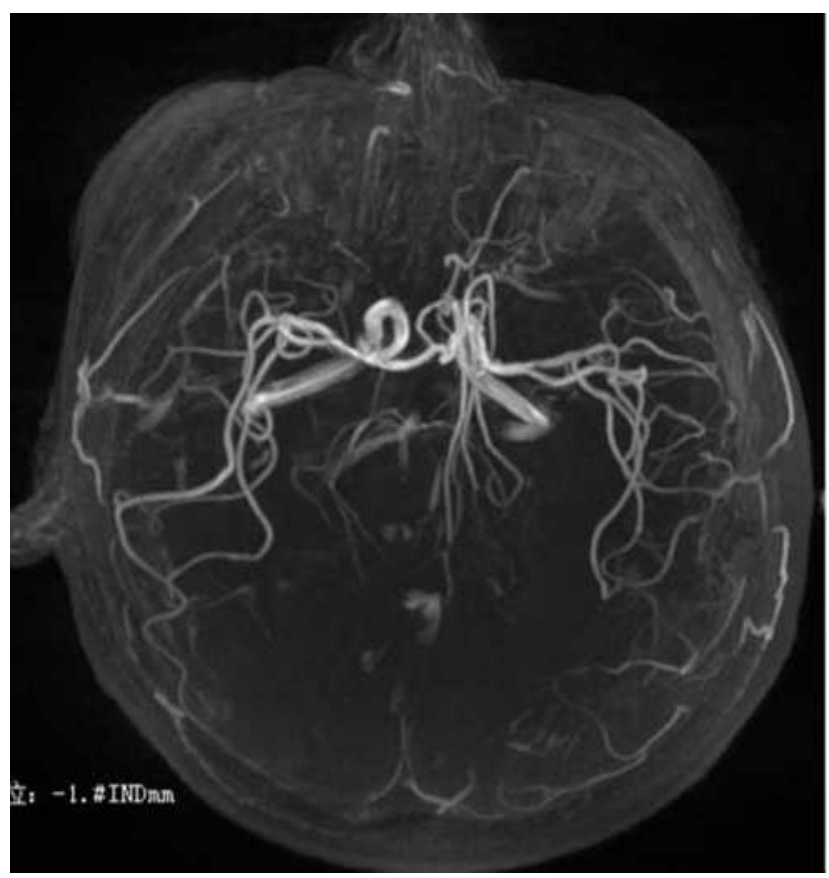

Figure 3 Magnetic resonance angiography demonstrating patent basilar artery and posterior cerebral arteries.

increased to 15 points after treatment. The MMSE scale score was 20 points (illiterate).

The patient's status was retrospectively reviewed one year after discharge from our hospital. The patient continued to have persistent cognitive dysfunction, memory decline, mental decline, vertical fixation paralysis, no fluency in speech and unstable emotions.

\section{Case 2}

A 57-year-old man was admitted to the emergency room after being unconscious for a day. One day earlier, the patient was found unconscious by his family. He was able to answer questions inaccurately but was unable to move his limbs and had slurred speech. The patient walked unsteadily and had difficulty urinating and had no vomiting nearby. The patient did not have limb stiffness or convulsion. Consciousness gradually worsened, and he was taken to the emergency department of the local county hospital approximately 3 hours later. Urgent head CT indicated bilateral hypodense lesions in the thalamus. The local county hospital considered the patient to have carbon monoxide toxic encephalopathy and was given hyperbaric oxygen therapy, and the consciousness disorder gradually aggravated. To obtain further diagnosis and treatment, the patient was sent to the Department of Neurology of our hospital by emergency department 120 . MRI examination was conducted immediately, which suggested bilateral thalamic acute infarction (Figure 4). He had no specific diseases in the past. He had no bad habits, such as smoking or drinking, no history of drugs or vaccination, and no history of exposure to toxic substances. Physical examination showed that the patient's consciousness was in a light coma. Neurological examination revealed that the patient could open his eyes after painful stimulation, the eyeballs stared upwards, and the eyeballs could be seen involuntarily rotating. The patient's bilateral pupils were equally large and round, with a diameter of approximately $3 \mathrm{~mm}$, light response was sensitive, no spontaneous nystagmus, tongue extension did not cooperate, active movement of the limbs was observed, unable to lift off the bed, normal muscle tension of the limbs, tendon reflex (++), and bilateral Babinski signs (-). The haematology test revealed the following: On an empty stomach, blood sugar was $8.51 \mathrm{mmol} / \mathrm{L}$, glycated 

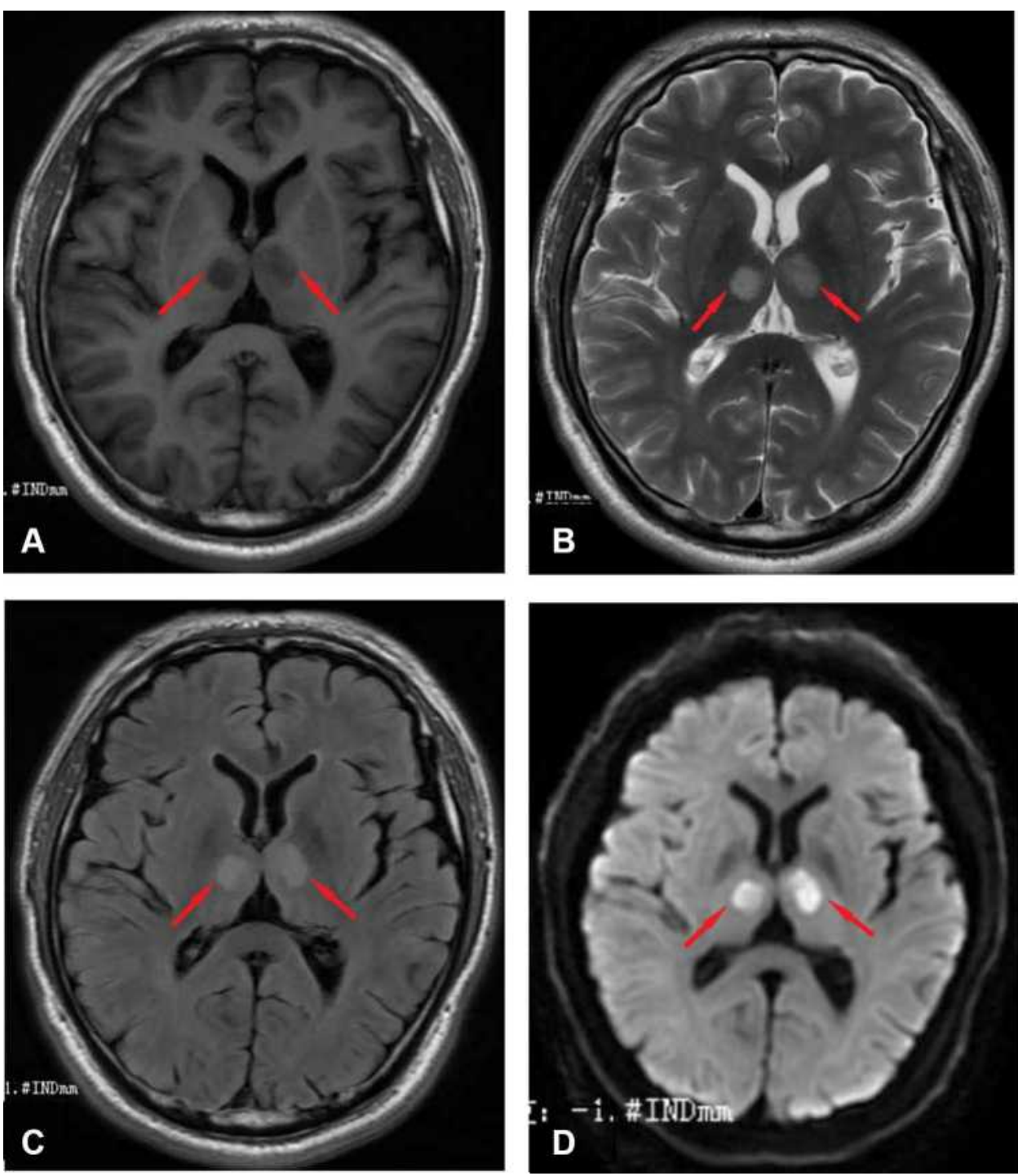

Figure 4 (A) TI* gradient echo MRI. Images show abnormal low signal bilateral paramedian thalamic lesions (arrows). (B) T2* gradient echo MRI. Images show abnormal hyperintense bilateral paramedian thalamic lesions (arrows). (C) Fluid attenuated inversion recovery echo MRI. Images show abnormal hyperintense bilateral paramedian thalamic lesions (arrows). (D) Diffusion-weighted axial MR showing diffusion restriction of the bilateral paramedian thalamus (arrows).

haemoglobin was $9.50 \%$, and low-density lipoprotein was $3.50 \mathrm{mmol} / \mathrm{L}$. Other haematology tests showed no abnormalities. The electrocardiogram was normal, echocardiography showed no abnormality, magnetic resonance angiography was normal (Figure 5), and digital subtraction angiography was normal. The patient gradually improved after 5 days in the hospital. During hospitalization, the patient was treated with blood sugar control, antiplatelet aggregation, plaque stabilization, circulation improvement, promotion of collateral circulation, elimination of oxygen free radicals and other treatments, and the clinical symptoms significantly improved. The patient was admitted to the hospital with a GCS score of 10 points, which increased to 15 points after treatment. The MMSE scale score was 27 points (secondary school level).

The patient was followed up one year after being discharged from the hospital, and he had poor memory and calculation ability.

\section{Discussion}

We described two cases in which MR imaging confirmed bilateral thalamic infarction. Bilateral thalamic infarction is relatively rare in clinical practice. The incidence of this type of cerebral infarction accounts for $0.1-2 \%$ of ischaemic cerebral infarction and $4-35 \%$ of all thalamic infarctions. ${ }^{7}$ The thalamus and the midbrain receive their 


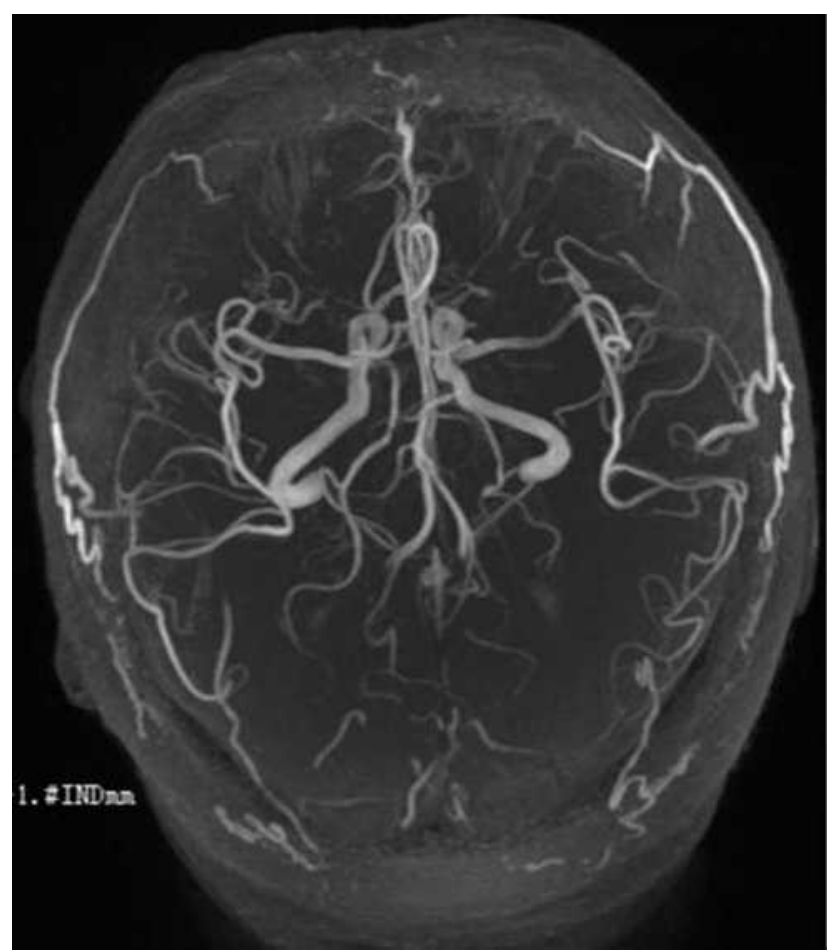

Figure 5 The basilar artery and bilateral posterior cerebral arteries were clearly seen by MR angiography.

blood mainly from the vertebrobasilar artery system, supplemented by the internal carotid artery system. ${ }^{1,2}$ If the artery of the Percheron is occluded, bilateral thalamic and mesencephalic infarctions occur. ${ }^{4}$ The Percheron artery was first proposed by French neurologist Percheron ${ }^{8}$ when describing the three variant types of the paramedian artery of the thalamus. Percheron arterial infarction mainly has acute onset, with varying degrees of consciousness disturbance, vertical gaze paralysis, memory impairment, etc. ${ }^{6} \mathrm{CT} / \mathrm{MRI}$ of the head showed paracentral bilateral thalamus infarction, sometimes combined with midbrain infarction. ${ }^{9}$

Currently, most researchers believe that the pathogenesis and causes of Percheron artery occlusion include: Small vessel disease (33-38.0\%), embolism (0-22\%), and large vessel disease (13.2-22.2\%). While embolism includes cardiogenic, arterial, and unexplained embolism, other causes include vasospasm secondary to subarachnoid haemorrhage, vascular dissection, hypercoagulable state, and inflammation of blood vessels secondary to central nervous system infection, such as tuberculous meningitis (13-15.7\%). ${ }^{10}$ We propose that when bilateral medial thalamic infarcts develop, occlusion of the artery of Percheron should be considered as the main diagnosis. Conventional angiography may not be indicated because lack of visualization of the artery does not exclude its presence (because it is occluded). ${ }^{4}$ Cerebral angiography should not be used for routine diagnosis of bilateral thalamic infarction caused by Percheron artery occlusion. When there are typical clinical manifestations and MRI changes, the presence of Percheron occlusion should be considered. ${ }^{9}$ Due to the difficulty in the diagnosis of acute Percheron arterial infarction, early conservative treatment is used. The prognosis of the disease is poor, with few patients completely rehabilitating. Most patients will experience sequelae, such as persistent cognitive dysfunction, memory decline, and intellectual and language dysfunction. ${ }^{11}$ Jodar et al $^{12}$ reported 2 cases of acute infarction of the Percheron artery treated with venous thrombosis. One patient was admitted to the hospital with a NIHSS score of 9 points, which dropped to 4 points after 24 hours of intravenous thrombosis, followed by a further drop to 1 point after 90 days. ${ }^{12}$ The other patient was admitted to the hospital with a NIHSS score of 21 points, which dropped to 1 point after 24 hours of venous lysis and 0 points after 90 days. ${ }^{12}$ Kostanian et al $^{13}$ reported a patient with Percheron arterial infarction in the acute stage who was treated by ultra-selective arterial thrombosis and cerebral angiostomy and showed that the Percheron artery was reconnected and that functional defects improved significantly. When diagnosing Percheron artery infarction, the identification of the following diseases should be considered: basilar artery apex syndrome, deep cerebral vein thrombosis, Wernicke encephalopathy, hypoxic ischaemic encephalopathy, hepatolenticular degeneration, osmotic myelinolysis, etc. Therefore, clinicians must fully understand the characteristics of Percheron arterial infarction and provide early diagnosis and timely effective treatment measures to reduce the patient's disability rate and fatality rate and therefore improve the quality of life of patients. The patient's prognosis has extraordinary significance.

\section{Ethics Approval}

This study was approved by the medical Ethics Committee of The First Hospital of Yulin. All the patients provided informed consent, and this study was conducted in accordance with the Declaration of Helsinki.

\section{Consent for Publication}

The institution was supportive of the publication of case details. 


\section{Acknowledgments}

We obtained consent for publication after discussion. Both case reports obtained patient consent, including publication of the images. We are grateful to the patient for his cooperation and support in preparing this case report.

\section{Funding}

This study was supported by the Technology Research and Development Project of Yulin City in 2019 ([2019] NO.185-42) and the Science and Technology Association Youth Talent Promotion Project Yulin City in 2020 (20200240). The funder was not involved in any part of this study, and the authors have no conflicts of interest to disclose.

\section{Disclosure}

The authors report no conflicts of interest in this work.

\section{References}

1. Percheron G. The anatomy of the arterial supply of the human thalamus and its use for the interpretation of the thalamic vascular pathology. Z Neurol. 1973;205:1-13. doi:10.1007/BF00315956

2. Lasjaunias P, Berenstein A, Brugge KGT, editors. Surgical Neuroangiography. 2nd ed. Vol. 1. Berlin: Springer-Verlag; 2000:526-562.
3. Schmahmann JD. Vascular syndromes of the thalamus. Stroke. 2003;34:2264-2278. doi:10.1161/01.STR.0000087786.38997.9E

4. Matheus MG. Imaging of acute bilateral paramedian thalamic and mesencephalic infarcts. AJNR Am $J$ Neuroradiol. 2003;24:2005-2008.

5. Caruso P, Manganotti P. Complex neurological symptoms in bilateral thalamic stroke due to Percheron artery occlusion. Vasc Health Risk Manag. 2017;13:11-14. doi:10.2147/VHRM.S119395

6. Ranasinghe T, Boo S, Adcock A. Acute onset of hypersomnolence and aphasia secondary to an artery of percheron infarct and a proposed emergency room evaluation. Case Rep Emerg Med. 2019;2019:1260865. doi:10.1155/2019/1260865.

7. Sandvig A, Lundberg S. Artery of Percheron infarction: a case report. J Med Case Rep. 2017;11:221. doi:10.1186/s13256-017-1375-3

8. Percheron G. Arteries of the human thalamus. I. Artery and polar thalamic territory of the posterior communicating artery. Rev Neurol (Paris). 1976;132(5):297-307.

9. Lin SF, Lin TC, Hu HH, et al. Bilateral paramedian thalamic infarction presenting as status epilepticus: a case report and review of the literatures. Acta Neurol Taiwan. 2015;24(4):125-130.

10. Xu Z, Sun L, Duan Y, et al. Assessment of Percheeron infarction in images and clinical findings. J Neurol Sci. 2017;383:87-92. doi:10.1016/j.jns.2017.10.033

11. Gentilini M, De Renzi E, Crisi G. Bilateral paramedian thalamic artery infarcts:report of eight cases. J Neurol Nerosurg Psychiatry. 1987;50(7):900-909. doi:10.1136/jnnp.50.7.900

12. Jodar M, Martos P, Fernández S, et al. Neuropsychological profile of bilateral paramedian infarctions: three cases. Neurocase. 2011;17 (4):345-352. doi:10.1080/13554794.2010.509324

13. Kostanian V, Cramer SC. Artery of percheron thrombolysis. Am J Neuroradiol. 2007;28(5):870-871.
Neuropsychiatric Disease and Treatment

\section{Publish your work in this journal}

Neuropsychiatric Disease and Treatment is an international, peerreviewed journal of clinical therapeutics and pharmacology focusing on concise rapid reporting of clinical or pre-clinical studies on a range of neuropsychiatric and neurological disorders. This journal is indexed on PubMed Central, the 'PsycINFO' database and CAS, and

\section{Dovepress}

is the official journal of The International Neuropsychiatric Association (INA). The manuscript management system is completely online and includes a very quick and fair peer-review system, which is all easy to use. Visit http://www.dovepress.com/testimonials.php to read real quotes from published authors. 